\title{
Monitoring of metabolism perturbation in prostate PC-3 cancer cells by sub-lethal concentrations of methotrexate
}

\author{
Allison Derenne, Régis Gasper and Erik Goormaghtigh* \\ Laboratory for the Structure and Function of Biological Membranes, Center for Structural Biology and \\ Bioinformatics, Université Libre de Bruxelles, Brussels, Belgium
}

\begin{abstract}
Infrared imaging was used to investigate the effect of sub-lethal concentration of methotrexate on prostate PC-3 cancer cells. Cells were exposed for 0,24 and $48 \mathrm{~h}$ to $9 \mathrm{nM}$ of methotrexate, the concentration that inhibits $50 \%$ of cell growth after $72 \mathrm{~h}$. Sub-lethal concentrations of methotrexate induced a reproducible signature that can be measured by infrared spectroscopy. Student $t$-test revealed that the process occurs in at least two steps, the first one affecting the nucleic acid region of the spectrum and the second one, the protein region. Imaging of individual cells demonstrated that the recorded differences are not due to a specific subpopulation of cells but homogenously affects all the cells. Near perfect identification of treated cells could be obtained using a supervised classification.
\end{abstract}

Keywords: IR spectroscopy, cancer, drug, IR imaging

\section{Abbreviations}

IR: Infrared

FTIR: Fourier Transform Infrared

h: Hours

S/N: Signal to Noise

PLS: Partial Least Square

\section{Introduction}

Infrared spectroscopy has been used for years to characterize chemical and biochemical compounds. This technique monitors the global chemical composition of the sample. Since a cell contains thousands of proteins characterized by different structures, nucleic acids, a variety of lipids and sugars, the observed spectrum is a superimposition of the spectra of all cell constituents [10]. IR spectra account not only for the chemical nature of cell molecules but also for their conformations and are in particular very sensitive to protein conformation $[6,7,9]$. Whereas microarrays deal with variations in the expression level of

\footnotetext{
*Corresponding author: Dr. Erik Goormaghtigh, Laboratory for the Structure and Function of Biological Membranes, Center for Structural Biology and Bioinformatics, Campus Plaine CP206/02, Université Libre de Bruxelles, Bld du Triomphe 2, CP206/2, B1050 Brussels, Belgium. Tel.: +32 265053 86; Fax: +32 265053 82; E-mail: egoor@ulb.ac.be.
} 
genes, infrared spectroscopy addresses the question of the end products, i.e. proteins and consequences of protein activity (sugars, lipids, ...). FTIR spectroscopy has the advantage of being a low-cost and fast method which is very sensitive to all kinds of molecules present in the cell. It has already been demonstrated that FTIR spectrum of bacteria provides a unique fingerprint that allows classification of bacterial sub-species [13,14]. Furthermore, this approach has been used to differentiate tumor and normal cells and to separate tumor cell lines with different biological behaviour. For instance, in vivo aggressiveness and in vitro migration of glioma cell lines were successfully predicted from their IR spectra while no molecular biology technique was available for this purpose $[1,3]$. In a previous study, we have shown that IR spectroscopy is sensitive enough to characterize the time dependence of metabolic changes induced by ouabain, a compound belonging to the cardiotonic steroid family [4].

Antitumor drugs are known to interfere with cell metabolism according to different mechanisms. While high doses of drugs result in cell death, sub-lethal doses could induce physiological perturbations that are representative of their mode of action. In turn, infrared spectroscopy could provide us with a signature which is relevant of this mode of action. Recently, Draux et al. report that subtoxic doses of gemcitabine, an anti-tumor drug, can be monitored by FTIR spectroscopy [2]. In the present paper we demonstrate, using infrared imaging on prostate PC-3 cells, that low dose of methotrexate produces a FTIR spectral signature which is highly reproducible and could be characteristic of the particular mode of DNA synthesis inhibition induced by methotrexate.

\section{Materials and methods}

\subsection{Cell culture and treatment}

The human prostate cancer PC-3 (CRL-1435) cell line was obtained from the American Type Culture Collection (ATCC, Manassas, VA, USA) and was maintained according to the supplier's instructions. The cells were incubated at $37^{\circ} \mathrm{C}$ in sealed (airtight) Falcon plastic dishes (Nunc, Invitrogen SA, Merelbeke, Belgium) in a humidified atmosphere of $5 \% \mathrm{CO}_{2}$. The cells were kept in exponential growth in RPMI medium supplemented with $10 \%$ fetal bovine serum (FBS), $1 \%$ penicillin/streptomycin (an antibiotic/antimycotic solution) and $1 \%$ kanamycin to prevent mycoplasms. Cell culture medium and FBS were purchased from Gibco (Invitrogen, Merelbeke, Belgium). Penicillin/streptomycin and kanamycin solutions were provided by Sigma-Aldrich SA (Bornem, Belgium).

For FTIR spectroscopy, cells were detached by means of a five-minute treatment with trypsin/EDTA buffer (Gibco, Invitrogen SA, Merelbeke, Belgium). The reaction was stopped by adding $1 \mathrm{ml}$ of culture medium. The cells were pelleted by a 2-minute centrifugation $(300 \mathrm{~g})$, and washed three times in isotonic solution $(\mathrm{NaCl}, 0.9 \%)$ to ensure complete removal of trypsin and culture medium.

\subsection{FTIR spectroscopy}

$0.5 \mu \mathrm{l}$ of cell pellet re-suspended in about $10 \mu \mathrm{l}$ was deposited on the $\mathrm{BaF}_{2}$ plate. Then, the sample was quickly evaporated in $\mathrm{N}_{2}$ flux. The IR data were collected using a Hyperion 3000 IR imaging system (Bruker Optics, Ettlingen, Germany), equipped with a $64 \times 64$ Mercury Cadmium Telluride (MCT) Focal Plane Array (FPA) detector. The data were collected in transmission mode from sample regions of $170 \times 170 \mu \mathrm{m}^{2}$. Every individual element of the array detector covered an area of $2.7 \times 2.7 \mu \mathrm{m}^{2}$. The spectral resolution was set to $8 \mathrm{~cm}^{-1}$. One IR image resulted in 4096 spectra, each one being the average of 256 scans (ca 5 min recording). All the spectra were preprocessed as follows. The water 
vapour contribution was subtracted as described previously [5,8] with $1956-1935 \mathrm{~cm}^{-1}$ as reference peak. The spectra were then normalized for equal area between 1582 and $1492 \mathrm{~cm}^{-1}$ (Amide II peak). Finally, spectra were retained for further analysis when, on the Amide I and II region, the absorbances after scaling were superior to -80 and inferior to 550 absorbance units and when the Signal-to-Noise ratio was better than $450: 1$. This ratio was calculated using $1750-1480 \mathrm{~cm}^{-1}$ spectral range for signal and $2000-1900 \mathrm{~cm}^{-1}$ region for noise determination.

The processing of the spectra was carried out by means of specific software generated in our lab running under Matlab (Mathworks Inc.).

\section{3. $\mathrm{IC}_{50}$ determination}

Overall cell growth was assessed by means of the colorimetric MTT (3-[4,5-dimethylthiazol-2yl]diphenyltetrazolium bromide, Sigma, Bornem, Belgium) assay, as detailed elsewhere [11]. The cells were incubated for $72 \mathrm{~h}$ in the presence or the absence (controls) of the tested compounds. Drug concentrations ranged between $10^{-10}$ and $10^{-6} \mathrm{M}$ (with semi-log concentration increases). Experiments were carried out 18 times over at least 3 weeks.

\section{Results and discussion}

In a first step we measured the concentration inhibiting $50 \%$ of the cell growth after $72 \mathrm{~h}$, called here $\mathrm{IC}_{50}$. As this value strongly depends on cell lines and culture conditions, it was important to determine it on PC-3 cells with our culture settings. The $\mathrm{IC}_{50}$ value was found to be $9 \pm 0.44 \mathrm{nM}$. This concentration did not induce increased cell death and was used throughout the present study.

For all the experiments, cells were grown to sub-confluence. Methotrexate was added either $24 \mathrm{~h}$ or $48 \mathrm{~h}$ before sub-confluence was reached. Controls of untreated cells were always grown in parallel. In the course of this study a total of 37,700 spectra (18 images) passing the $\mathrm{S} / \mathrm{N}$ threshold as described in Section 2 were recorded for untreated cells. Similarly 4900 spectra ( 2 images) from cells incubated for $24 \mathrm{~h}$ in the presence of methotrexate and 21,300 spectra (7 images) from cells exposed for $48 \mathrm{~h}$ to the drug were obtained.

Student $t$-tests: In order to determine the wavenumbers at which the absorbance was significantly different between non-treated and $24 \mathrm{~h}$ incubation in the presence of methotrexate or between $24 \mathrm{~h}$ and $48 \mathrm{~h}$ exposure to the drug, a Student $t$-test was performed at every wavenumber with a significance $\alpha=0.5 \%$. Figure 1 reports differences between the mean spectra for all the data collected after $24 \mathrm{~h}$ incubation in the presence and absence of methotrexate. Wavenumbers where a significant difference occurred are indicated by stars. It can be observed on Fig. 1 that most of significant differences are located between 1300 and $1000 \mathrm{~cm}^{-1}$, a spectral region usually assigned to nucleic acids [12], with little effect in the protein region of the spectrum $\left(1700-1500 \mathrm{~cm}^{-1}\right)[6,12]$. The $\mathrm{C}-\mathrm{H}$ stretching region of the spectrum (3050-2800 $\mathrm{cm}^{-1}$ ) did not display any significant difference (not shown). The difference between the mean spectra obtained at 48 and $24 \mathrm{~h}$ incubation is also reported in Fig. 1. A simple examination of this spectrum indicates that this second difference has fewer significant modifications in the nucleic acid region but display a marked change in the protein region which is completely new with respect to the alterations observed in the first $24 \mathrm{~h}$. At this stage of the research it is not possible to speculate on the molecular origin of this difference as, even if dominated by proteins, different types of molecules can absorb in this region [12]. Yet, the results obtained suggest that the metabolic perturbations induced by methotrexate proceed in at least two steps, the first one affecting the nucleic acid 


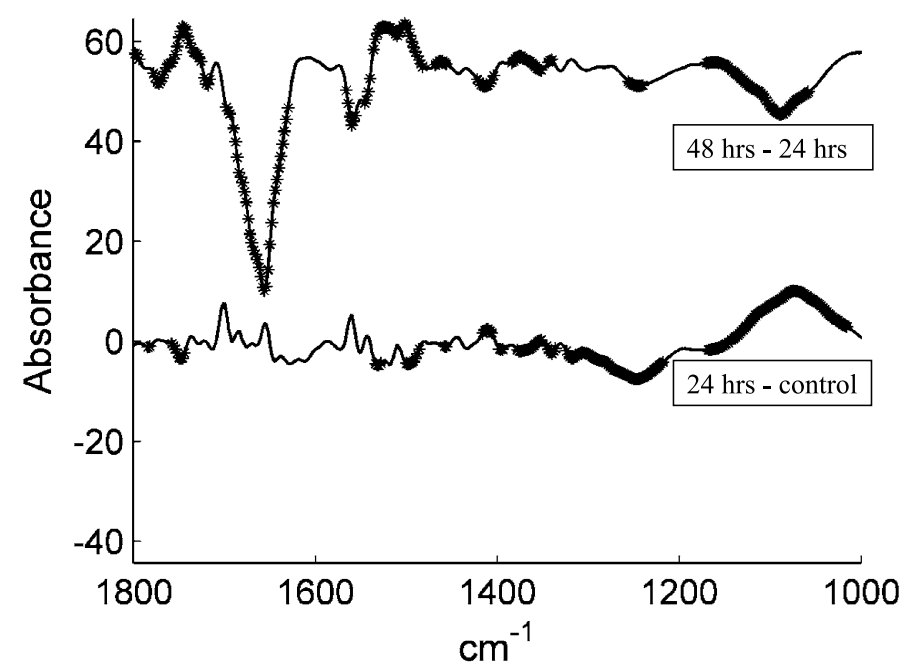

Fig. 1. Difference between the mean spectra of cells exposed to methotrexate for $24 \mathrm{~h}$ and untreated cells (bottom) and difference between the mean spectra of cells exposed to 48 and $24 \mathrm{~h}$ to methotrexate (top). Methotrexate concentration was $9 \mathrm{nM}$. Before computing the means, spectra were corrected for water vapor absorption and were rescaled so that the amide II area between 1582 and $1492 \mathrm{~cm}^{-1}$ was set to an arbitrary equal value for all the spectra. A Student $t$-test $(\alpha=0.5 \%)$ was computed at every wavenumber and, when the difference between the means was found to be significant, a star was placed on the difference spectrum.

region of the spectrum and the second one the protein region. Again the $\mathrm{C}-\mathrm{H}$ region dominated by the lipids was not significantly affected (not shown). Importantly, Student $t$-tests comparing several control experiments on untreated cells could not evidence any significant difference. This was also confirmed by principal component analysis, an unsupervised approach which, in our hands, easily detects problems with controls (e.g., infection by mycoplasms).

Imaging: The results of the Student $t$-test suggest that a clear distinction can be obtained between untreated cells and cells exposed for $24 / 48 \mathrm{~h}$ to $\mathrm{IC}_{50}$ concentration of methotrexate. The question imaging can address is how the changes observed on the means are shared among the different cells visible on a microscopic field. Figure 2 reports two microscopic fields fused in one single image. The first one originates from untreated cells and the second one from cells exposed for $48 \mathrm{~h}$ to methotrexate. Taking advantage of the large variation between the mean spectra detected on Fig. 1 in the protein region near $1656 \mathrm{~cm}^{-1}$, the image simply reports the absorbance at this latter wavenumber. Interestingly, it appears that all the cells from the sample are affected in a similar way. It can be concluded that there is a single cell population with a homogenous response to methotrexate.

Discriminant PLS: As a single wavenumber allows a good separation between cells exposed to methotrexate and controls, a supervised classification such as obtained by discriminant PLS should achieve an even better identification of the state of the cells. Experiments (not shown) indeed revealed that $99.7 \%$ of the spectra were classified correctly when a validation test was considered, i.e. the prediction was run on a series of cells which did not participate to the construction of the PLS model $\left(1800-1000 \mathrm{~cm}^{-1}\right)$.

In conclusion, we have shown here that sub-lethal concentrations of methotrexate induce a reproducible signature which can be measured in prostate PC-3 cancer cells. Student $t$-test emphasizes that the process occurs in two steps, the first one affecting the nucleic acid region of the spectrum and the second one, the protein region. Imaging of individual cells demonstrates that the recorded differences 


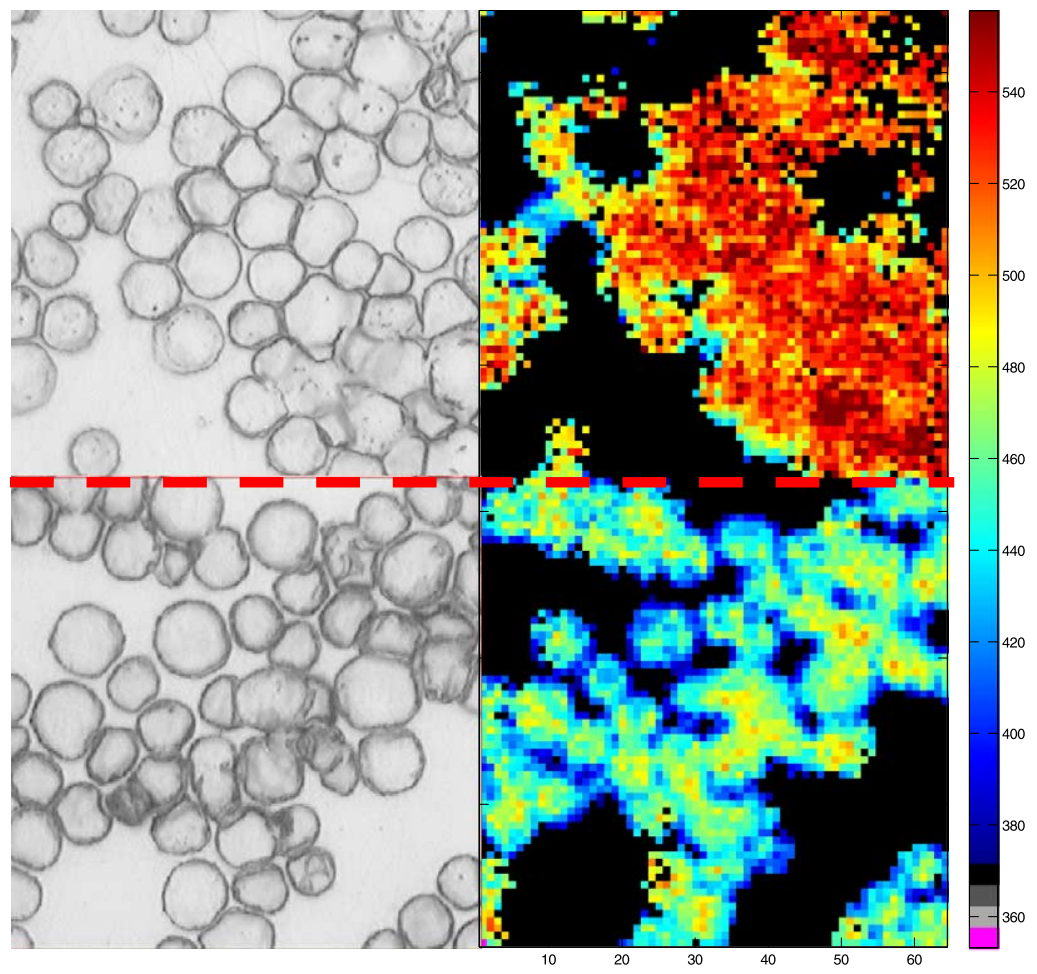

Fig. 2. Visible (left column) and color-coded absorbance at $1656 \mathrm{~cm}^{-1}$ (right column) of prostate PC-3 cancer cells. The upper half of each column displays cells that were exposed to methotrexate $(9 \mathrm{nM})$ for $48 \mathrm{~h}$, the lower half displays untreated cells. Spectra have been corrected for water vapor and scaled as explained in Section 2 and in Fig. 1. The areas of the image where the signal/noise ratio was below threshold (450) are black.

is not due to a subpopulation of cells but homogenously affects all the cells. Finally, nearly perfect identification of treated cells can be obtained using a supervised classification.

\section{Acknowledgements}

This research has been supported by a grant from Interuniversity Attraction Poles (IAP) P6/19 (Belgium) and the National Fund for Scientific Research (FRFC 2.4533.10 and 2.4588.06). Erik Goormaghtigh is Directors of Research with the "National Fund for Scientific Research", Belgium. Allison Derenne is supported by the FRIA (Belgium).

\section{References}

[1] M.A. Cohenford and B. Rigas, Cytologically normal cells from neoplastic cervical samples display extensive structural abnormalities on IR spectroscopy: implications for tumor biology, Proc. Natl. Acad. Sci. USA 95 (1998), 15327-15332.

[2] F. Draux, P. Jeannesson, C. Gobinet et al., IR spectroscopy reveals effect of non-cytotoxic doses of anti-tumour drug on cancer cells, Anal. Bioanal. Chem. (2009).

[3] A. Gaigneaux, C. Decaestecker, I. Camby et al., The infrared spectrum of human glioma cells is related to their in vitro and in vivo behavior, Exp. Cell Res. 297 (2004), 294-301.

[4] R. Gasper, J. Dewelle, R. Kiss, T. Mijatovic and E. Goormaghtigh, IR spectroscopy as a new tool for evidencing antitumor drug signatures, Biochim. Biophys. Acta 1788 (2009), 1263-1270. 
[5] E. Goormaghtigh, FTIR data processing and analysis tools, in: Advances in Biomedical Spectroscopy, Vol. 2, A. Barth and P.I. Haris, eds, IOS Press, Amsterdam, 2009, pp. 107-128.

[6] E. Goormaghtigh, V. Cabiaux and J.M. Ruysschaert, Determination of soluble and membrane protein structure by Fourier transform infrared spectroscopy. III. Secondary structures, Subcell. Biochem. 23 (1994), 405-450.

[7] E. Goormaghtigh, R. Gasper, A. Benard, A. Goldsztein and V. Raussens, Protein secondary structure content in solution, films and tissues: redundancy and complementarity of the information content in circular dichroism, transmission and ATR FTIR spectra, Biochim. Biophys. Acta 1794 (2009), 1332-1343.

[8] E. Goormaghtigh and J.M. Ruysschaert, Subtraction of atmospheric water contribution in Fourier transform infrared spectroscopy of biological membranes and proteins, Spectrochim. Acta 50A (1994), 2137-2144.

[9] E. Goormaghtigh, J.M. Ruysschaert and V. Raussens, Evaluation of the information content in infrared spectra for protein secondary structure determination, Biophys. J. 90 (2006), 2946-2957.

[10] C. Matthaus, B. Bird, M. Miljkovic, T. Chernenko, M. Romeo and M. Diem, Infrared and Raman microscopy in cell biology, Methods Cell Biol. 89 (2008), 275-308.

[11] T. Mijatovic, D.B. Op, E. van Quaquebeke et al., The cardenolide UNBS1450 is able to deactivate nuclear factor kappaBmediated cytoprotective effects in human non-small cell lung cancer cells, Mol. Cancer Ther. 5 (2006), 391-399.

[12] D. Naumann, Infrared spectroscopy in microbiology, in: Encyclopedia of Analytical Chemistry, R.A. Meyers, ed., Wiley, Chichester, 2000, pp. 102-131.

[13] D. Naumann, D. Helm and H. Labischinski, Microbiological characterizations by FT-IR spectroscopy, Nature 351 (1991), $81-82$.

[14] C.A. Rebuffo, J. Schmitt, M. Wenning, F. von Stetten and S. Scherer, Reliable and rapid identification of Listeria monocytogenes and Listeria species by artificial neural network-based Fourier transform infrared spectroscopy, Appl. Environ. Microbiol. 72 (2006), 994-1000. 


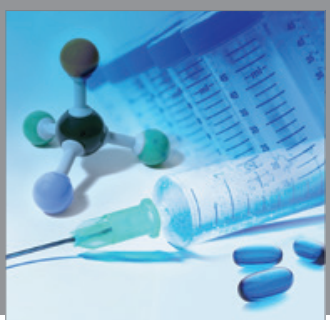

International Journal of

Medicinal Chemistry

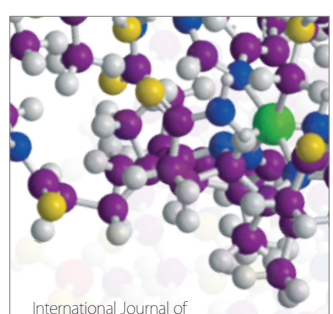

Carbohydrate Chemistry

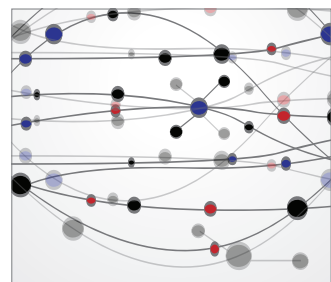

The Scientific World Journal
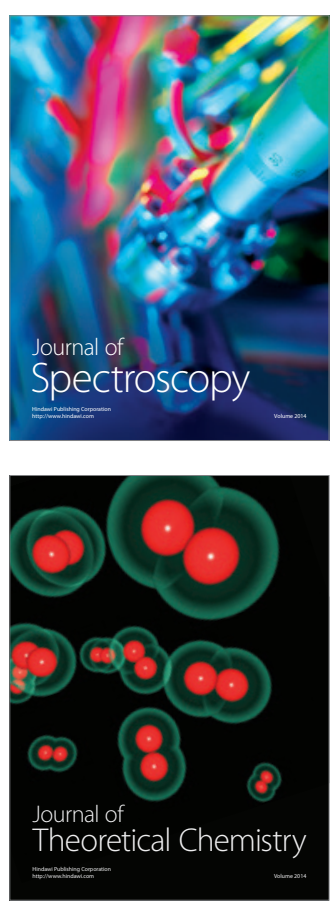
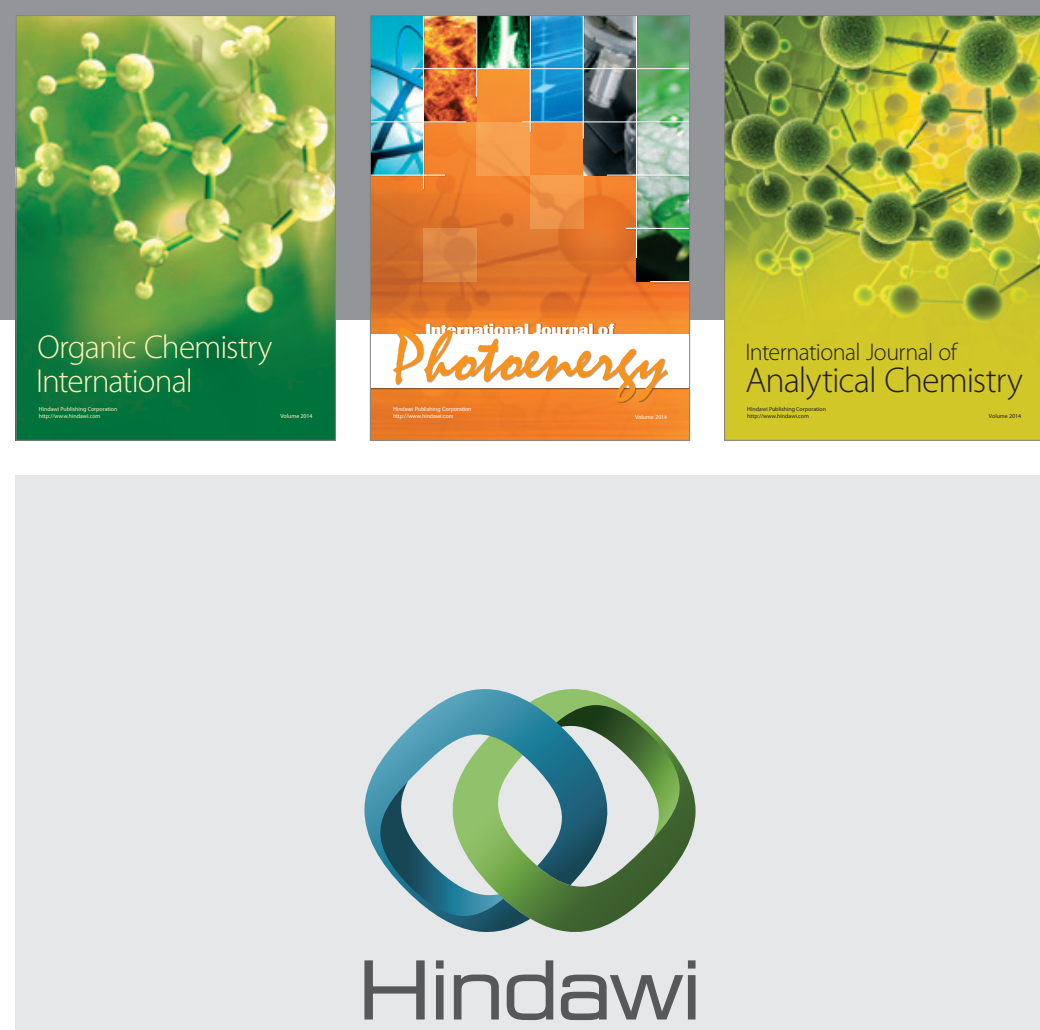

Submit your manuscripts at

http://www.hindawi.com
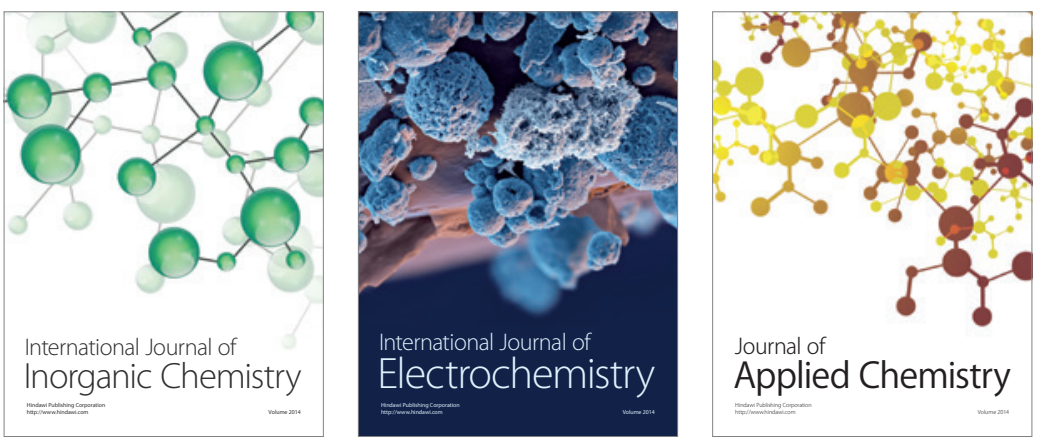

Journal of

Applied Chemistry
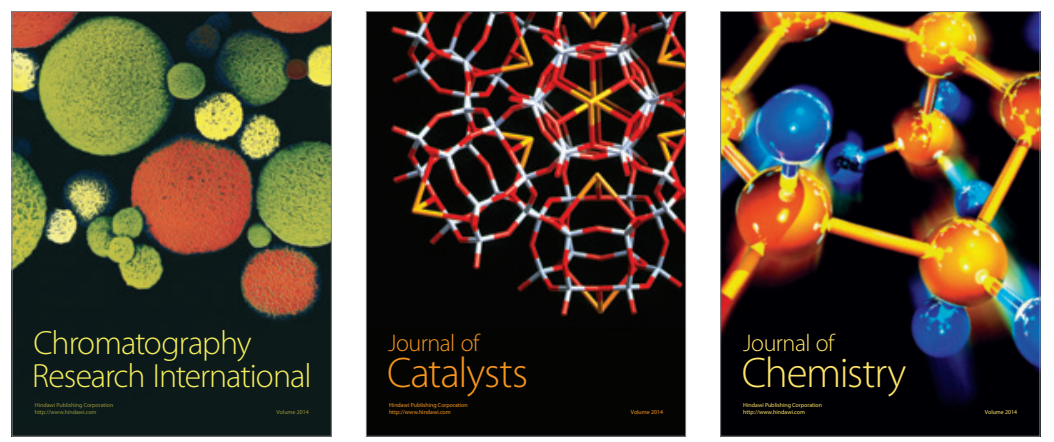
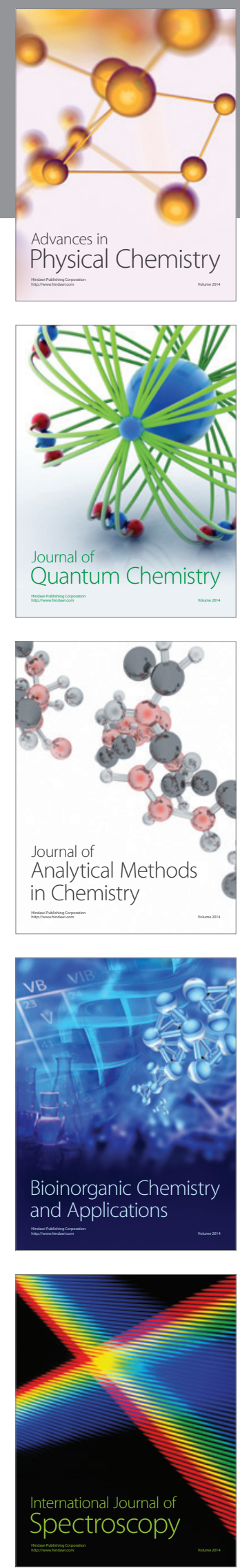